\title{
Erratum to: A FAS-ligand variant associated with autoimmune lymphoproliferative syndrome in cats
}

\author{
Danielle Aberdein $^{1} \cdot$ John S. Munday $^{1} \cdot$ Barbara Gandolfi $^{2} \cdot$ Keren E. Dittmer $^{1} \cdot$ \\ Richard Malik $^{3}$ - Dorian J. Garrick ${ }^{1,4} \cdot$ Leslie A. Lyons $^{2} \cdot 99$ Lives Consortium
}

Published online: 18 January 2017

(C) Springer Science+Business Media New York 2017

\section{Erratum to: Mamm Genome 28(1-2):47-55 DOI 10.1007/s00335-016-9668-1}

The work on FAS-ligand included a dataset that had 83 cats - not 54 cats. Attached is the corrected consortium list for those who contributed cat sequences and resources for the 99 Lives Consortium of 83 cats.

\section{Appendix}

\section{Lives Consortium (54 Cat database)}

Funding for the 99 Lives Cat Genome Sequencing Initiative has been provided by the Winn Feline Foundation and the George Sydney and Phyllis Redman Miller Trust (MT-13010), the National Geographic Society Education Foundation (2P-14), the University of Missouri Gilbreath-McLorn Endowment (LAL), contributions from the 99 Lives

The online version of the original article can be found under doi:10.1007/s00335-016-9668-1.

John S. Munday

j.munday@massey.ac.nz

1 Pathobiology, Institute of Veterinary, Animal, and Biomedical Sciences, Massey University, Palmerston North, New Zealand

2 Department of Veterinary Medicine and Surgery, College of Veterinary Medicine, University of Missouri - Columbia, Columbia, MO 65211, USA

3 Centre for Veterinary Education, University of Sydney, Sydney, NSW 2006, Australia

4 Department of Animal Science, Iowa State University, Ames, IA 50011, USA
Consortium participants, donations from Associazione Nazionale Felina Italiana, Zoetis, Orivet Genetic Pet Care, Langford Veterinary Services, the World Cat Federation, and public donations. We appreciate the provision of cat DNA samples by Cristy Bird, Bruno Chomel, Kyung Sik Kim, Nassem N. Naimi of Best Friend Veterinary Clinic in Amman, Jordan, Julie Pomerantz, John Snape, Susanne and Claus Wehnert, Nancy Carpenter at Utah's Hogle Zoo, Ashleigh Lutz-Nelson at San Francisco Zoo \& Gardens and Julie Feinstein at the American Museum of Natural History

Pauol C. Alves ${ }^{4-6}$, Gregory S. Barsh ${ }^{7,8}$, Holly C. Beale ${ }^{9}$, Adam R. Boyko ${ }^{10}$, Marta G. Castelhano ${ }^{11}$, Patricia Chan ${ }^{9}$, N. Matthew Ellinwood ${ }^{12}$, Christopher R. Helps ${ }^{13}$, Christopher B. Kaelin ${ }^{7,8}$, Tosso Leeb ${ }^{14}$, Hannes Lohi ${ }^{15}$, Maria Longeri $^{16}$, Michael J. Montague ${ }^{17}$, William J. Murphy ${ }^{18}$, Niels C. Pedersen ${ }^{19}$, Max F. Rothschild ${ }^{12}$, William F. Swanson $^{20}$, Karen A. Terio ${ }^{21}$, Rory J. Todhunter ${ }^{11}$, Wesley C. Warren ${ }^{18}$.

${ }^{4} \mathrm{CIBIO} / \mathrm{InBIO}$, Centro de Investigação em Biodiversidade e Recursos Genéticos, Universidade do Porto, Campus Agrário de Vairão, 4485-661, Vairão, Portugal.

${ }^{5}$ Departamento de Biologia, Faculdade de Ciências da Universidade do Porto (FCUP), 4169-007 Porto, Portugal.

${ }^{6}$ Wildlife Biology Program, University of Montana, Missoula, Montana, 59812 USA.

${ }^{7}$ HudsonAlpha Institute for Biotechnology, Huntsville, Alabama 35806, USA.

${ }^{8}$ Department of Genetics, Stanford University, Stanford, California, 94305 USA.

${ }^{9}$ Maverix Biomics, Inc., San Mateo, California, 94402 USA.

${ }^{10}$ Department of Biomedical Sciences, College of Veterinary Medicine, Cornell University, Ithaca, New York, 14853 USA. 
${ }^{11}$ Department of Clinical Sciences, College of Veterinary Medicine, Cornell University, Ithaca, New York, 14853 USA.

${ }^{12}$ Department of Animal Science, College of Agriculture and Life Sciences, Iowa State University, Ames, Iowa, 50011 USA.

${ }^{13}$ Langford Veterinary Services, University of Bristol, Langford, Bristol, BS40 5DU UK.

${ }^{14}$ Vetsuisse Faculty, Institute of Genetics, University of Bern, Bern, Switzerland. Swiss Competence Center of Animal Breeding and Genetics, University of Bern, 3001, Bern University of Applied Sciences HAFL \& Agroscope, 3001, Bern, Switzerland.

${ }^{15}$ Department of Veterinary Biosciences and Research Programs Unit, Molecular Neurology, University of Helsinki and Folkhälsan Research Center, Helsinki 00014, Finland.

${ }^{16}$ Dipartimento di Scienze Veterinarie e Sanità Pubblica, University of Milan, 20122 Milan, Italy.

${ }^{17}$ The Genome Institute, Washington University School of Medicine, St. Louis, Missouri, 63108 USA.

${ }^{18}$ Department of Veterinary Integrative Biosciences, College of Veterinary Medicine, Texas A\&M University, College Station, Texas, 77845 USA.

${ }^{19}$ Department of Medicine and Epidemiology, School of Veterinary Medicine, University of California at Davis, Davis, California, 95616 USA.

${ }^{20}$ Center for Conservation and Research of Endangered Wildlife (CREW), Cincinnati Zoo \& Botanical Garden, Cincinnati, Ohio, 45220 USA.

${ }^{21}$ Zoological Pathology Program, University of Illinois, Maywood, IL 60153.

\section{Lives Consortium (83 cat analysis)}

Leslie A. Lyons ${ }^{1}$, Danielle Aberdein ${ }^{2}$, Paulo C. Alves ${ }^{3,4}$, Gregory S. Barsh ${ }^{5,6}$, Holly C. Beale ${ }^{7}$, Adam R. Boyko ${ }^{8}$, Jeffrey A. Brockman ${ }^{9}$, Marta G. Castelhano ${ }^{10}$, Patricia P. Chan ${ }^{7}$, N. Matthew Ellinwood ${ }^{11}$, Jonathan E. Fogle ${ }^{12}$, Dorian J. Garrick ${ }^{2,11}$, Christopher R. Helps ${ }^{13}$, Marjo K. Hytönen $^{14}$, Maria Kaukonen ${ }^{14}$, Christopher B. Kaelinn ${ }^{5,6}$, Emilie Leclerc ${ }^{15}$, Tosso Leeb ${ }^{16}$, Hannes Lohi ${ }^{14}$, Maria Longeri ${ }^{17}$, Richard Malik ${ }^{18}$, Michael J. Montague ${ }^{19}$, John S. Munday $^{2}$, William J. Murphy ${ }^{20}$, Niels C. Pedersen ${ }^{21}$, Max F. Rothschild ${ }^{11}$, Joshua A. Stern ${ }^{21}$, William F. Swanson ${ }^{22}$, Karen A. Terio ${ }^{23}$, Rory J. Todhunter ${ }^{10}$, Yu Ueda ${ }^{21}$, Wesley C. Warren ${ }^{19}$, Elizabeth A. Wilcox ${ }^{10}$, Julia H. Wildschutte ${ }^{24}$, Barbara Gandolfi ${ }^{1}$.

${ }^{1}$ Department of Veterinary Medicine and Surgery, College of Veterinary Medicine, University of Missouri, Columbia, Missouri, 65211 USA.
${ }^{2}$ Institute of Veterinary, Animal and Biomedical Sciences, Massey University, Palmerston North 4474 New Zealand.

${ }^{3} \mathrm{CIBIO} / \mathrm{InBIO}$, Centro de Investigação em Biodiversidade e Recursos Genéticos/InBIO Associate Lab \& Faculdade de Ciências, Universidade do Porto, Campus e Vairão, 4485 - 661 Vila do Conde, Portugal.

${ }^{4}$ Wildlife Biology Program, University of Montana, Missoula, Montana, 59812 USA.

${ }^{5}$ HudsonAlpha Institute for Biotechnology, Huntsville, Alabama, 35806 USA.

${ }^{6}$ Department of Genetics, Stanford University, Stanford, California, 94305 USA.

${ }^{7}$ Maverix Biomics, Inc., San Mateo, California, 94402 USA.

${ }^{8}$ Department of Biomedical Sciences, College of Veterinary Medicine, Cornell University, Ithaca, New York, 14853 USA.

${ }^{9}$ Hill's Pet Nutrition Inc., PO Box 1658, Topeka, KS 66601 USA.

${ }^{10}$ Department of Clinical Sciences, College of Veterinary Medicine, Cornell University, Ithaca, New York, 14853 USA.

${ }^{11}$ Department of Animal Science, College of Agriculture and Life Sciences, Iowa State University, Ames, Iowa, 50011 USA.

${ }^{12}$ College of Veterinary Medicine, North Carolina State University, Raleigh, NC 27607.

${ }^{13}$ Langford Veterinary Services, University of Bristol, Langford, Bristol, BS40 5DU UK.

${ }^{14}$ Department of Veterinary Biosciences and Research Programs Unit, Molecular Neurology, University of Helsinki and Folkhälsan Research Center, Helsinki 00014 Finland.

${ }^{15}$ Diana Pet food, Inc. SPF - ZA du Gohelis, 56250 Elven, France.

${ }^{16}$ Vetsuisse Faculty, Institute of Genetics, University of Bern, 3001 Bern, Switzerland.

${ }^{17}$ Dipartimento di Medicina Veterinaria, University of Milan, 20122 Milan, Italy.

${ }^{18}$ Centre for Veterinary Education, University of Sydney, Sydney, NSW, 2006 Australia.

${ }^{19}$ The McDonnell Genome Institute, Washington University School of Medicine, St. Louis, Missouri, 63108 USA.

${ }^{20}$ Department of Veterinary Integrative Biosciences, College of Veterinary Medicine, Texas A\&M University, College Station, Texas, 77845 USA.

${ }^{21}$ Department of Medicine and Epidemiology, School of Veterinary Medicine, University of California at Davis, Davis, California, 95616 USA. 
${ }^{22}$ Center for Conservation and Research of Endangered Wildlife (CREW), Cincinnati Zoo \& Botanical Garden, Cincinnati, Ohio, 45220 USA.

${ }^{23}$ Zoological Pathology Program, University of Illinois, Brookfield, IL 60513.
${ }^{24}$ Bowling Green State University, Department of Biological Sciences, Bowling Green, OH 43403. 